\title{
Severe symptoms of short tear break-up time dry eye are associated with accommodative microfluctuations
}

This article was published in the following Dove Press journal:

Clinical Ophthalmology

5 May 2017

Number of times this article has been viewed

\author{
Minako Kaido ${ }^{1,2}$ \\ Motoko Kawashima' \\ Reiko Ishida ${ }^{1,3}$ \\ Kazuo Tsubota' \\ 'Department of Ophthalmology, \\ Keio University School of Medicine, \\ Tokyo, ${ }^{2}$ Wada Eye Clinic, Chiba, \\ ${ }^{3}$ Ishida Eye Clinic, Shizuoka, Japan
}

Aim: Validating the hypothesis that accommodative microfluctuations (AMFs) may be associated with severe symptoms in short tear break-up time (BUT) dry eye (DE).

Methods: This study included 12 subjects with short BUT DE (age: $49.6 \pm 18.3$ years). Diagnoses were performed based on the presence of DE symptoms, BUT $\leq 5 \mathrm{~s}$, Schirmer score $>5 \mathrm{~mm}$, and negative keratoconjunctival epithelial damage. Tear evaluation, AMF, and functional visual acuity (VA) examinations were conducted before and after DE treatment. The AMF parameters evaluated were: total high-frequency component (HFC), HFC with low accommodation for the task of staring into the distance (HFC1), HFC with high accommodation for deskwork (HFC2). A subjective questionnaire of DE symptoms was also performed.

Results: Mean BUT increased from $1.9 \pm 2.0$ to $6.4 \pm 2.5 \mathrm{~s}$ after treatment $(P<0.05)$. The mean logarithm of the minimum angle of resolution functional VA significantly improved (from $0.19 \pm 0.19$ to $0.12 \pm 0.17 ; P<0.05)$. Mean power spectrum values for total HFC and HFC1 decreased (from $61.3 \pm 5.7$ to $53.8 \pm 6.6 \mathrm{~dB}$ and from $62.9 \pm 10.5$ to $52.4 \pm 6.2 \mathrm{~dB}$, respectively; $P<0.05$ ), while the mean HFC2 power spectrum values did not differ before and after treatment $(P>0.05)$. Subjective DE symptoms were reduced in nine patients.

Conclusion: Along with the improvement of BUT after treatment, DE symptoms diminished and HFC1 and functional VA improved, suggesting that tear film instability is associated with deterioration of functional VA, AMF, and DE symptoms.

Keywords: accommodative microfluctuation, ciliary muscle spasm, dry eye, ocular fatigue, tear break-up time, functional visual acuity

\section{Introduction}

The rapid rise in the prevalence of dry eye (DE) disease has been a public health issue over the last few years, ${ }^{1-4}$ particularly in a highly technological and informationoriented society with the spread of Internet at a worldwide level. Visual display terminal (VDT) exposure is becoming common in the general population because of the widespread use of mobile technology and portable information terminals, particularly smartphones, not only among young people, but also among the middle-aged and the elderly.

Recent studies revealed that VDT-related DE has some distinct characteristics, such as the presence of DE symptoms and tear film instability despite a normal lacrimal secretion, also known as the short tear break-up time (BUT) DE. ${ }^{4-6}$ This type of DE is rising in today's society because of the widespread use of computers. In fact, the Osaka study, which is a Japanese survey of DE prevalence associated with VDT work, revealed that $\sim 75 \%$ VDT users had a shortened BUT value. ${ }^{4,5}$ Short BUT DE
Correspondence: Minako Kaido Department of Ophthalmology, Keio University School of Medicine, Shinanomachi 35, Shinjuku-ku,

Tokyo 160-8582, Japan

Tel +81353633269

Fax +8I 333598302

Email fwiwl193@mb.infoweb.ne.jp
Clinical Ophthalmology 2017:1 I 861-869 (c) (1) (\$) $\odot 2017$ kaido et al. This work is published and licensed by Dove Medical Press Limited. The full terms of this license are available at https://www.dovepress.com/terms.php

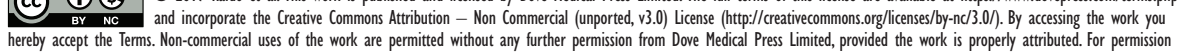

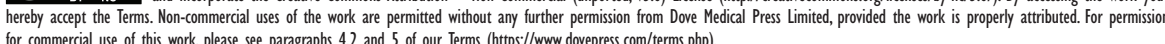


is diagnosed based not only on tear film instability, but also on the presence of symptoms. However, the presence of deficiency in tear secretion and corneal epithelial damage are not absolutely necessary. Accordingly, short BUT $\mathrm{DE}$ is sometimes regarded as a mild, or vague, type of DE. Instead, patients with short BUT DE often suffer from severe DE symptoms, which are similar to those of aqueous teardeficient DE with corneal epithelial damage. ${ }^{7,8}$ Reports of subjective symptoms of short BUT DE include ocular fatigue (rather than dryness), while aqueous-deficient DE patients mainly complain of foreign body sensation and dryness. ${ }^{7-9}$ So far, it is not known why short BUT DE presents ocular fatigue as a typical symptom.

It is thought that ocular fatigue is associated with accommodation. ${ }^{10}$ In this accommodative response, rapid and continuous fluctuations are known to occur when the eye views a target. ${ }^{11}$ Fluctuations are composed of a low-frequency component (LFC) of $<0.6 \mathrm{~Hz}$ and a high frequency component (HFC) between 1.0 and $2.3 \mathrm{~Hz} .{ }^{11}$ Interestingly, it has been reported that ocular fatigue is related to HFC. ${ }^{12-14}$ Here, we proposed and assessed the hypothesis that accommodative microfluctuations (AMFs) may be associated with DE symptoms in short BUT DE.

\section{Methods}

\section{Patients}

Twelve short BUT DE patients (five men, seven women; mean age: $49.6 \pm 18.3$ years; age range: $23-77$ years), treated at the DE subspecialty outpatient clinic at the Department of Ophthalmology at Keio University, were enrolled in this study. These patients had severe DE symptoms and/or a history showing that ordinary DE treatments were not effective in reducing their symptoms. Table 1 shows the profile of symptoms, baseline clinical signs, and treatments. The eye with more severe symptoms, or the right eye when the symptoms were equal in both eyes, was studied. Short BUT DE was diagnosed by the presence of DE symptoms, a BUT value $\leq 5 \mathrm{~s}$, and a Schirmer test score $>5 \mathrm{~mm}$ with no positive fluorescein staining scores (staining scores $<3$ points). Subjects were excluded if they had a history of ocular trauma, abnormality of the nasolacrimal drainage apparatus, disorder of ocular movement, or wore contact lenses. The patients with systemic diseases including hormonal imbalance were also excluded.

This research followed the tenets of the Declaration of Helsinki. Informed consent was obtained from all subjects after explanation of the nature and possible consequences of the study. Ethics committee approval for the examination procedures and study protocol were obtained from Keio University (Ethics Approval Number at Keio University: 2013-144).

\section{Tear function and ocular surface evaluation}

Tear stability was assessed by standard BUT measurement after instillation of a $2 \mu \mathrm{L}$ volume of a preservative-free solution of $1 \%$ fluorescein in the conjunctival sac with a micropipette. BUT was measured three times, and the mean value of the measurements was calculated. Keratoconjunctival epithelial damage was evaluated based on the Japanese DE diagnostic criteria, using the method of van Bijsterveld. ${ }^{15}$ To evaluate tear quantity, the Schirmer test without anesthesia was performed following all other examinations, using a sterilized Schirmer strip (Whatman No 41; Showa Yakuhin Kako Co., LTD, Tokyo, Japan).

\section{The functional visual acuity measurement system}

We performed continuous visual acuity (VA) testing during a $60 \mathrm{~s}$ period under natural blinking, using the Functional VA Measurement System (Kowa, Aichi, Japan). The outcome parameters were: starting VA, functional VA, visual maintenance ratio, and blink frequency. ${ }^{5}$ Patients were asked to describe the orientation of automatically presented Landolt rings by manipulation of a joystick.

\section{AMF analysis system}

The AMF test was performed using a dynamic autorefractometer (AA-2 ${ }^{\mathrm{TM}}$; NIDEK Co., Ltd, Nagowa, Japan). The target was presented on an open-field refractometer. Based on the refraction at the far distance, targets were set from +0.5 to $-3.0 \mathrm{D}$ of objective refraction in steps of $0.5 \mathrm{D}$ (ie, eight steps in total). The patients were instructed to maintain a clear gaze at the target in each step. The refractive error, range of accommodation, and AMF were measured. According to the AMF values, the power spectrum of HFC was calculated by fast Fourier transformation as the frequency of HFC appearance, converting the values to the common logarithm and summating the values between 1.0 and $2.25 \mathrm{~Hz} .{ }^{16}$ The graph (Fk-map: frequency of kinetic reaction-map) appears in spectral colors representing the values of the frequency of HFC appearance.

The results of the power spectrum of HFC were divided into two parameters: HFC1 with low accommodation (between 0.0 and $0.75 \mathrm{D}$ accommodation constriction), for the task of staring into the distance, and HFC2 with high accommodation 


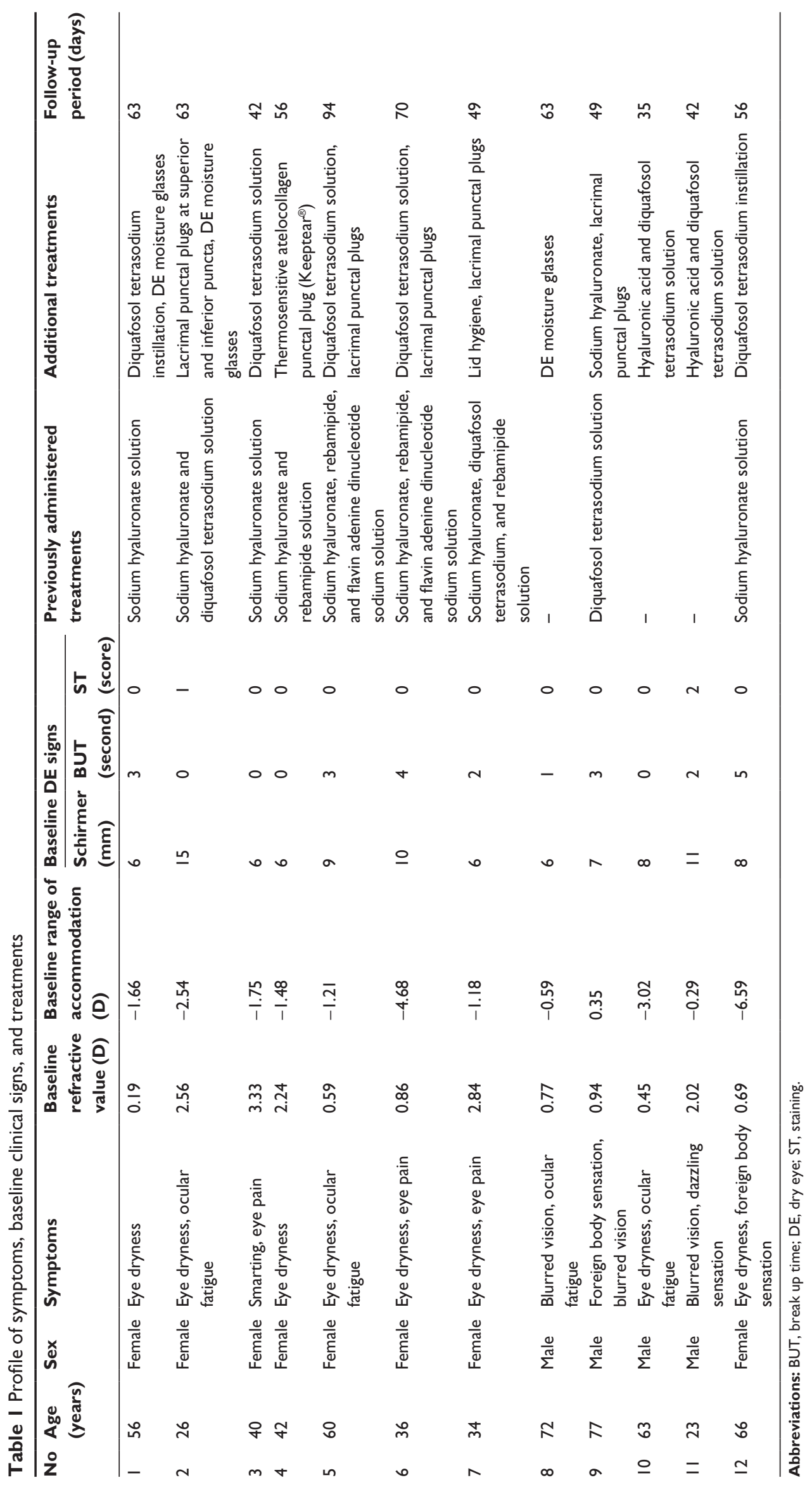


(between -1.0 and -3.0 D), for deskwork. Total HFC was defined as the summation of the power spectrum of HFC, with accommodation between 0.75 and $-3.0 \mathrm{D}$.

The evaluation parameters measured by the autorefractometer were: refraction at far distance, range of accommodation gazing at the target loading between 0.75 and $-3.0 \mathrm{D}$, the power spectrum of HFC1, HFC2, and total HFC.

\section{Treatment protocol}

DE treatments were provided depending on the prescriptions of the patients, as shown in Table 1.

\section{Questionnaire analysis of subjective DE symptoms}

We asked about one of the most severe DE-related symptoms. A Verbal Rating Scale was used for the assessment of symptomatic improvement after treatment. There were four degrees of improvement of symptoms: "great improvement" (3 points), "moderate improvement" (2 points), "slight improvement" (1 point), and "no improvement" (0 points).

\section{Statistical analysis}

Tear function, functional VA parameters, and accommodative parameters were compared before and after treatment, using a paired $t$-test. SPSS software version $17.0 \mathrm{~J}$ for Windows (SPSS Inc, Chicago, IL, USA) was used for statistical analysis. A $P$-value of $<0.05$ was considered statistically significant.

\section{Results}

The patients were checked for the curative effect and compliance every month. The follow-up periods ranged from 35 to 94 days (mean: $56.8 \pm 15.7$ days). Nine patients $(75.0 \%)$ showed improvement of their symptoms after treatment. More specifically, slight, moderate, or great improvement of ocular symptoms after treatment was reported by five $(41.7 \%)$, two (16.7\%), and two (16.7\%) patients, respectively, while no improvement was reported by three patients $(25.0 \%)$.

\section{Tear function assessment}

The mean BUT value significantly increased from 1.9 \pm 1.7 to $6.4 \pm 2.5 \mathrm{~s}(P<0.05)$, whereas the fluorescein scores before and after treatment were $0.7 \pm 0.8$ and $0.4 \pm 1.0$ points, respectively, and did not show a significant difference $(P>0.05)$.

\section{Functional VA parameters}

Figure $1 \mathrm{~A}-\mathrm{C}$ shows data for the functional VA parameters before and after treatment. The mean logarithm of the minimum angle of resolution of functional VA significantly improved $(P<0.05)$, while the mean logarithm of the minimum angle of resolution of conventional VA did not show any statistically significant difference before and after treatment $(P>0.05)$. The mean visual maintenance ratio had a tendency to improve $(P=0.06)$.

\section{Refractive and accommodative parameters}

The mean refractive values before and after treatment were $-2.05 \pm 1.95$ and $-1.97 \pm 1.59 \mathrm{D}$, respectively. No significant difference was observed between these values $(P>0.05)$. The mean range of accommodation before and after treatment was $1.46 \pm 1.07$ and $1.38 \pm 0.97 \mathrm{D}$, respectively, and no significant difference was observed between these values $(P>0.05)$.

Figure 1D shows the mean values of the power spectrum for HFC1, HFC2, and total HFC before and after treatment. The mean values of the power spectrum for HFC1 and total HFC significantly decreased $(P<0.05)$, while the mean value of the power spectrum for HFC2 did not show any statistically significant difference before and after treatment $(P>0.05)$.

Figures 2 and 3 show the representative cases.

\section{Discussion}

Short BUT DE is characterized by the presence of tear film instability and DE symptoms. ${ }^{7,9}$ Typical aqueous-deficient DE symptoms are dryness and foreign body sensation. However, the symptoms of short BUT DE often include nonspecific complaints such as "ocular fatigue", ${ }^{9}$ and these symptoms are often very severe. Therefore, we conducted this study to elucidate why short BUT DE patients suffer from severe DE symptoms, despite normal lacrimal secretion and lack of corneal damage.

We have previously reported visual deterioration in short BUT DE patients, using functional VA measurements. ${ }^{17}$ Indeed, we often observe a large visual fluctuation over time during measurement of functional VA in short BUT DE, while the pattern of visual changes in aqueous teardeficient DE includes a sudden drop in VA. Similar to previous studies, our results showed that functional VA was relatively deteriorated before treatment, but it improved along with the symptoms and BUT value after treatment. On the other hand, conventional VA did not show any difference before and after treatment. We also found that short BUT $\mathrm{DE}$ showed a high value of the power spectrum for HFC of $\mathrm{AMF}$, and that the value of the power spectrum for HFC was decreased after treatment aimed at maintaining tear stability. 
A

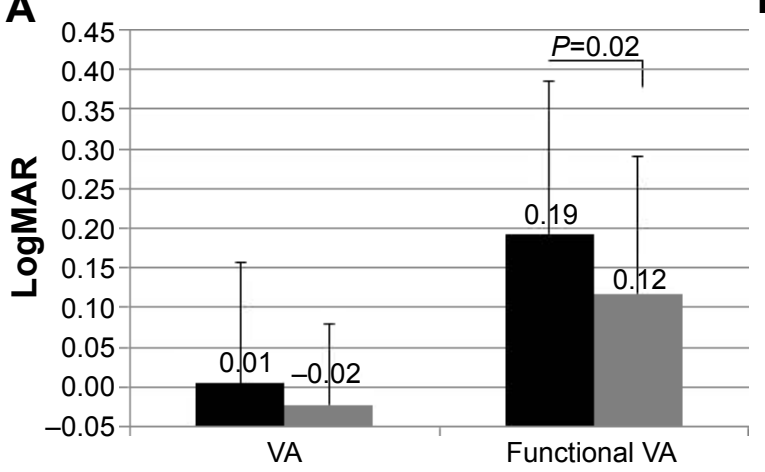

B

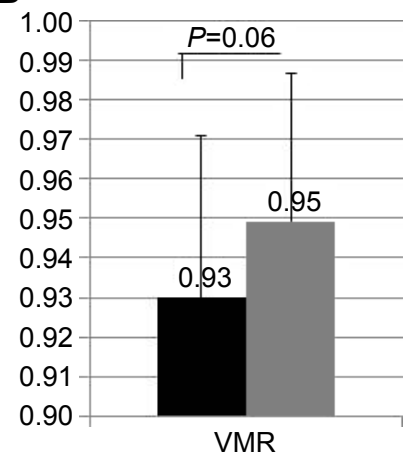

C

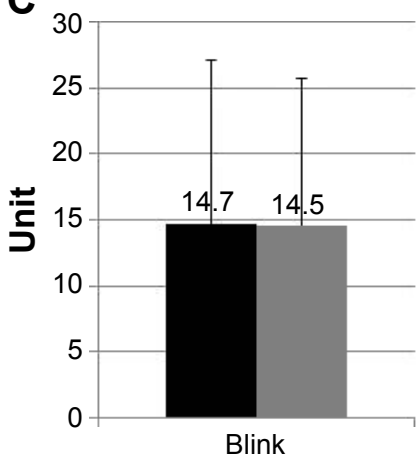

D

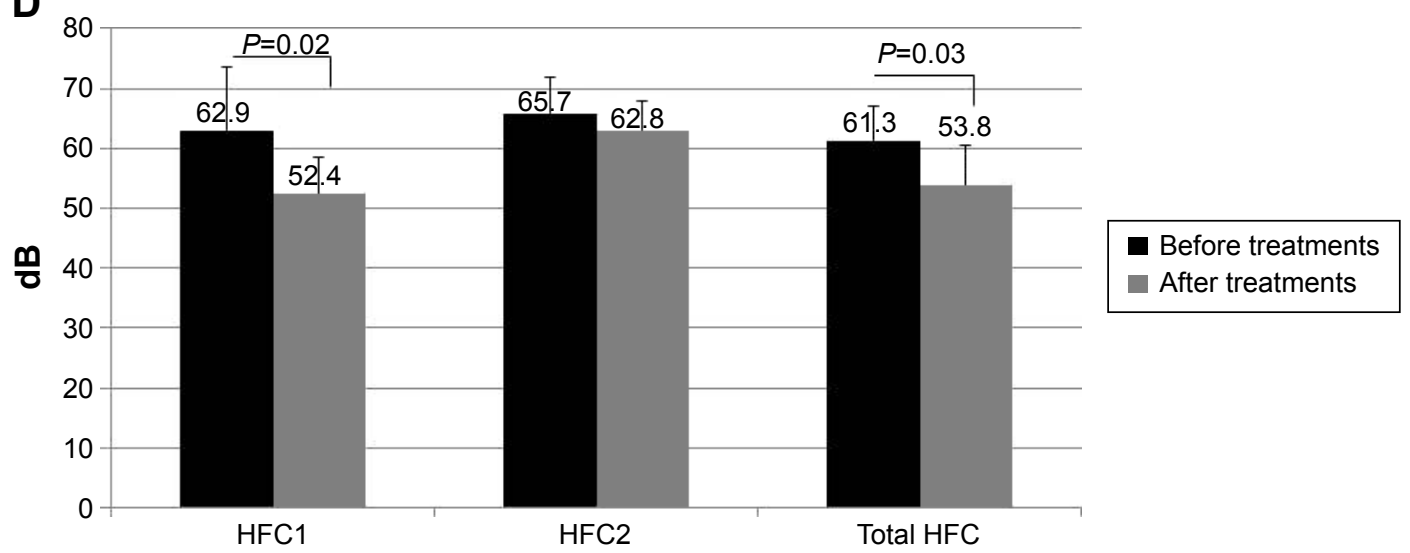

Figure I Functional VA and accommodative parameters before and after additional treatments.

Notes: (A) Starting VA and functional VA: Starting VA was defined as the standard best-corrected VA, as measured by the Functional VA Measurement System; functional VA was defined as the average of all VA values measured over time. LogMAR of functional VA significantly improved $(P<0.05)$, while the mean logMAR of conventional VA did not show any statistically significant difference before and after treatment $(P>0.05)$. (B) Visual maintenance ratio: Visual maintenance ratio was defined as the functional VA divided by the starting VA. (C) Blink frequency: The blink frequency was automatically recorded by a functional VA measurement device. (D) Accommodative parameters. The mean values of the power spectrum for HFCI and total HFC significantly decreased $(P<0.05)$, while the mean value of the power spectrum for $\mathrm{HFC2}$ did not show any statistically significant difference before and after treatment $(P>0.05)$.

Abbreviations: HFC, high-frequency component; logMAR, logarithm of the minimum angle of resolution; VA, visual acuity; VMR, visual maintenance ratio.

Taking into account the presence of both visual deterioration and high values of the power spectrum for HFC of AMF in short BUT DE, tear film instability may be associated with symptoms of short BUT DE.

A major cause of ocular fatigue is related to accommodation..$^{10}$ Kajita et al reported that power spectrum values for HFC were higher in subjects with asthenopia, compared to normal subjects without any eye-related symptoms. ${ }^{12}$ Moreover, they found that among the AMF parameters, there was an association between ocular fatigue and HFC1, but not HFC2 ${ }^{12}$ In this study, we found similar results in the sense that power spectrum values for HFC1 were relatively high before treatment, but decreased as the DE symptoms resolved, along with the improvement of BUT after treatment. On the other hand, the power spectrum value for HFC2 did not show any difference before and after treatment. Interestingly, this phenomenon was observed also in elderly patients with presbyopia; a decrease in accommodation ability of the eye due to an alteration of the lens flexibility.
As part of the HFC function, HFC2 is related to microfluctuations when the eye views a target at a near distance, while HFC1 plays a role in far distance with a low burden accommodation. Accordingly, regarding the strain of accommodation, we would expect $\mathrm{HFC} 2$ to play a role in DE symptoms, rather than HFC1. However, the results revealed that only HFC1 was associated with DE symptoms. This phenomenon may not be induced by a direct strain on the ciliary muscles, which are necessary for accommodation to the distant and near focus, because $\mathrm{HFC} 1$ is related to microfluctuations when viewing a target at a far distance and high HFC1 was also observed in elderly patients with presbyopia.

It is known that instability and irregularity of the tear film may cause visual fluctuations and deterioration. ${ }^{17,18}$ Indeed, in this study, we found that functional VA tests assessing far distance vision showed a fluctuation and decrease over time in short BUT DE. Blurring may be due to ocular and/or periocular stress, possibly caused by ciliary muscle spasms. In young patients, the lens may fluctuate due to ciliary muscle spasm, 
A

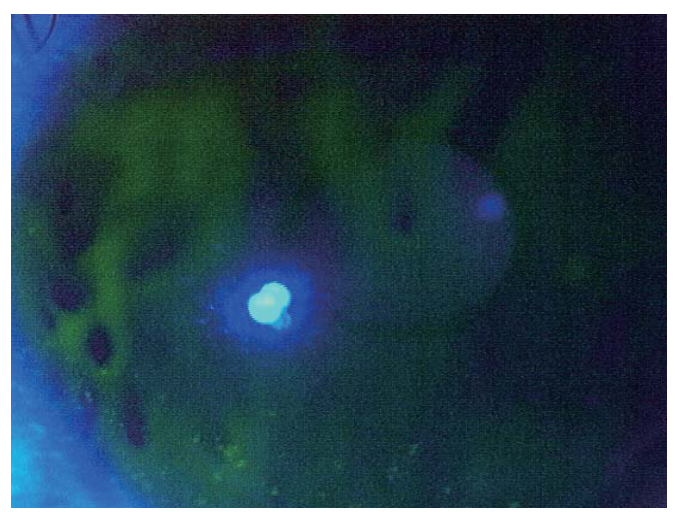

B

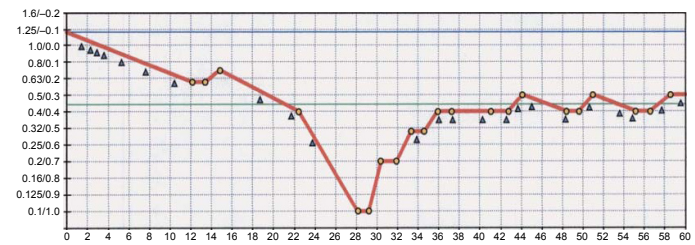

C

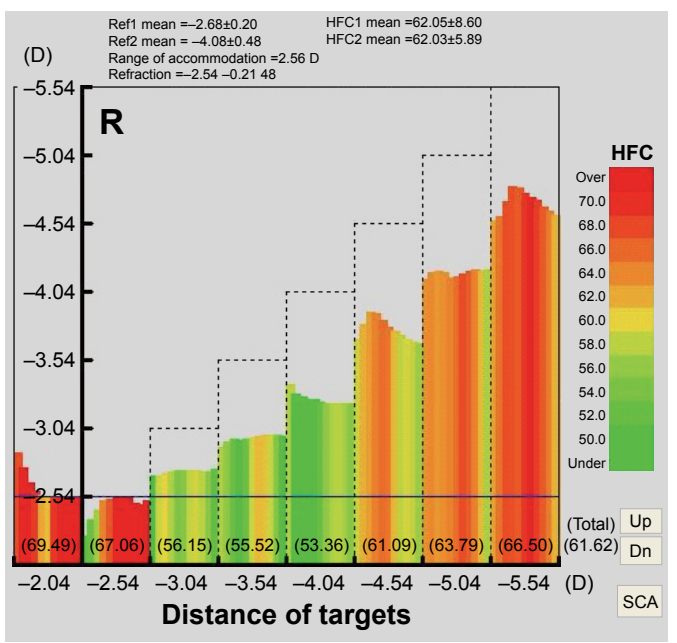

D

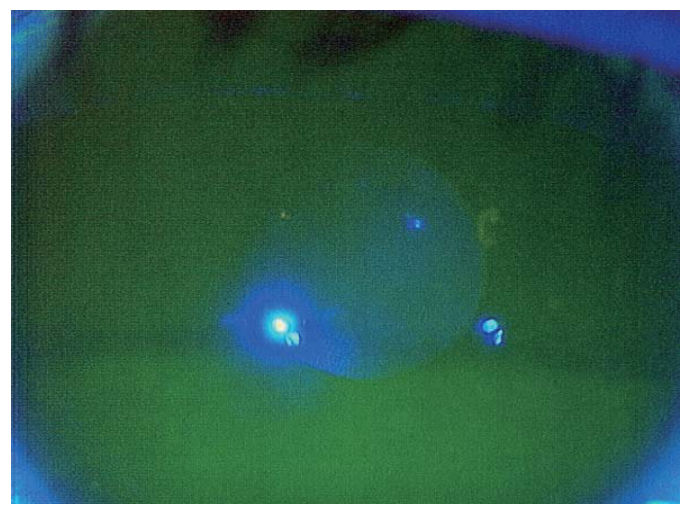

E

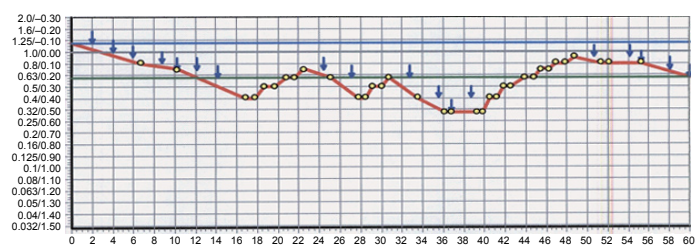

$\mathbf{F}$

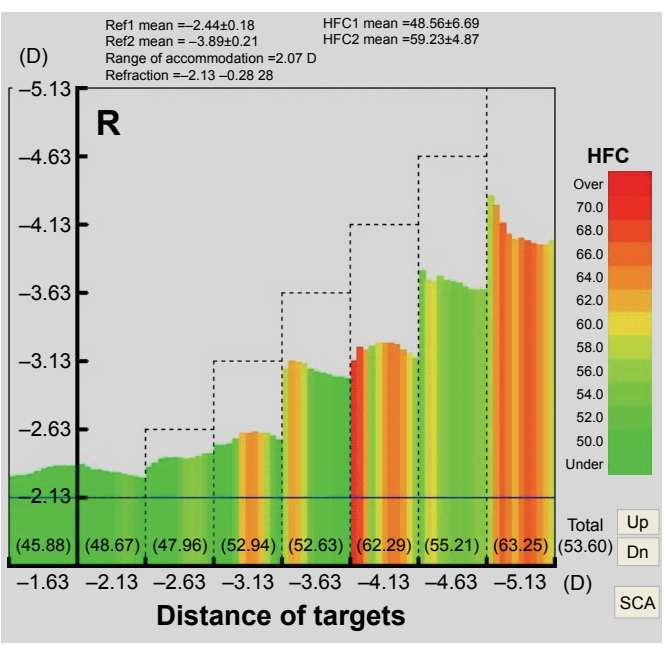

Figure 2 Fk-map and visual changes over time in a young patient.

Notes: (A) Tear film on the ocular surface before additional treatments. (B) Functional VA before the additional treatments. (C) Fk-map before the additional treatments. (D) Tear film on the ocular surface after the additional treatments. (E) Functional VA after the additional treatments. (F) Fk-map after the additional treatments. A 26year-old woman (Case No 2) suffered from DE which was unresponsive to treatment. The BUT value was $0 \mathrm{~s}$ (A). Visual large fluctuation (B) and high appearance of HFCI (C) were observed. Two months after the additional treatment consisting of insertion of lacrimal plugs to the superior and inferior lacrimal puncta and wearing DE moisture glasses, the DE symptoms completely resolved. The BUT value improved to $9 \mathrm{~s}(\mathbf{D})$. Likewise, functional VA (E) and accommodative parameters (F) improved.

Abbreviations: BUT, break-up time; DE, dry eye; Fk-map, frequency of kinetic reaction-map; HFC, high-frequency component; VA, visual acuity.

which eventually induces HFC1 and causes DE symptoms including ocular fatigue. On the other hand, the phenomenon of HFC1 values being relatively high before treatment and reducing as the DE symptoms are resolved after treatment was also observed in elderly patients with presbyopia. Why was a fluctuation in HFC1 induced in elderly patients, even though the crystalline lens is no longer flexible? What is the potential cause for the rhythmic fluctuation of HFC1? A ciliary muscle spasm may be induced even when the lens flexibility is feeble. A ciliary muscle spasm might shift the position of the crystalline lens back and forth, or waves of the ciliary muscle spasm may propagate to the bulbus oculi. Such motion consequently may induce a rhythmic fluctuation in the depth of the anterior chamber (ie, axial length), inducing the fluctuation of HFC1, regardless of the functional status of the crystalline lens (ie, whether the lens retains its flexibility or not). Thus, the fluctuation of HFC1 may be observed in both young and elderly patients. Figure 4 shows the mechanism of occurrence of DE symptoms in short BUT DE. In fact, it has been reported that pseudo-accommodation can be produced by drug-induced ciliary muscle contraction, even in eyes with intraocular lenses. ${ }^{16}$ This supports that a fluctuation in the lens may be induced by ciliary muscle spasm in eyes with presbyopia. Moreover, it has been reported that additional treatment with a low-density-level cyclopentolate was effective for short BUT DE. ${ }^{19}$ Our results support that HFC1 may 
A

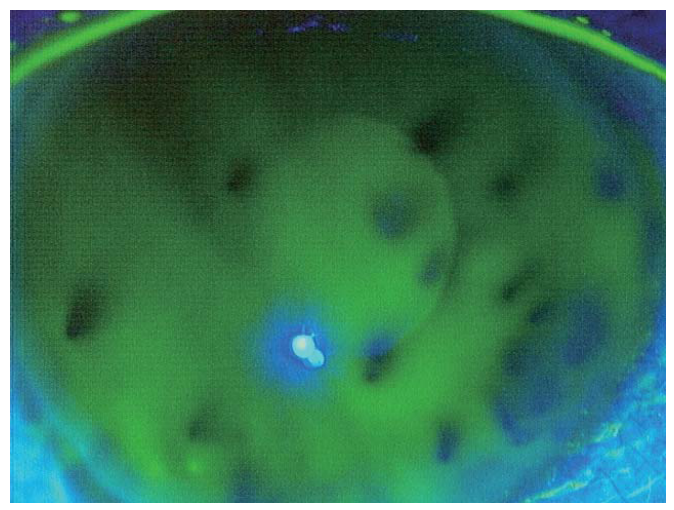

B

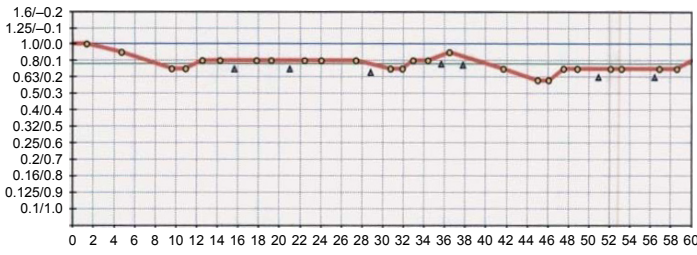

C

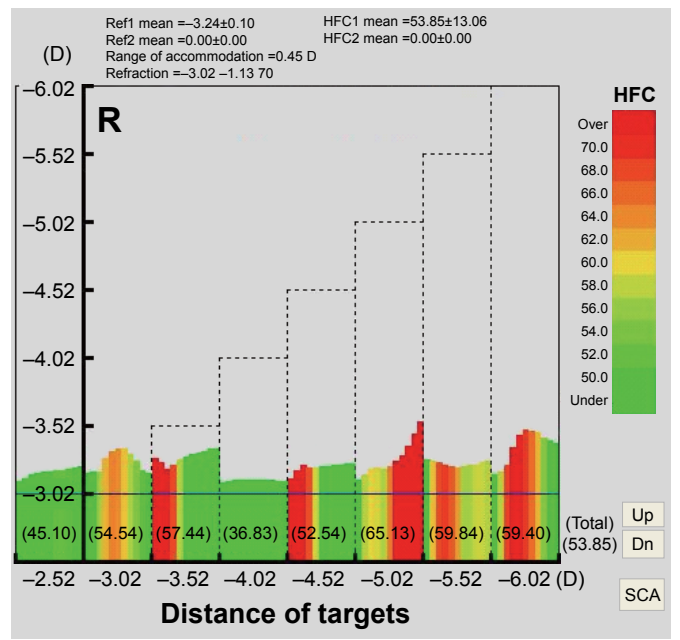

D

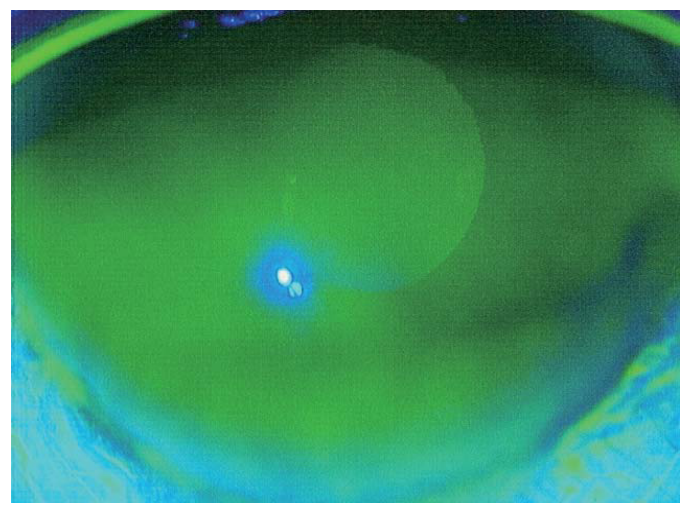

E

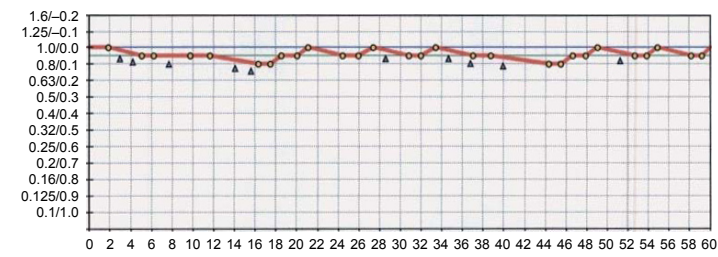

$\mathbf{F}$

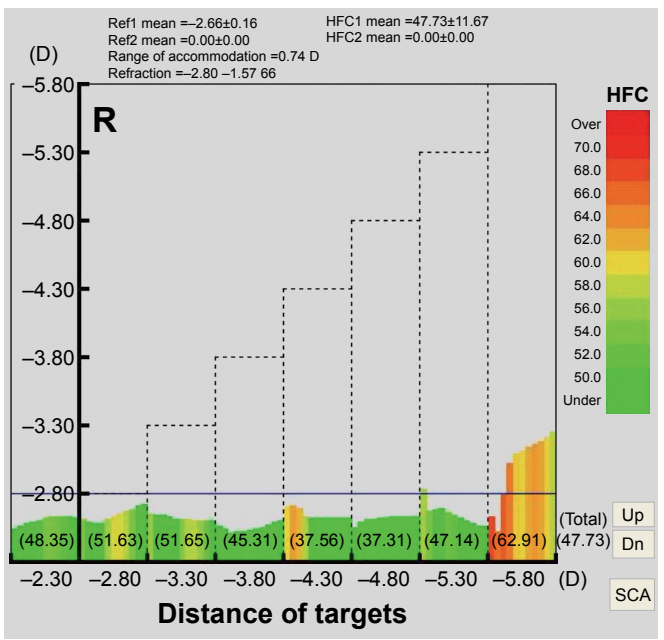

Figure 3 Fk-map and visual changes over time in an elderly patient.

Notes: (A) Tear film on the ocular surface before additional treatments. (B) Functional VA before the additional treatments. (C) Fk-map before the additional treatments. (D) Tear film on the ocular surface after the additional treatments. (E) Functional VA after the additional treatments. (F) Fk-map after the additional treatments. A 63-yearold man (Case No I0) suffered from severe DE sensation and ocular fatigue over the past years. A previously performed lacrimal plug insertion in the lacrimal puncta was maladaptive, and was removed because of foreign body sensation. The Fk-map showed a pattern of presbyopia (B). After topical administration of $0.1 \%$ sodium hyaluronate and $3 \%$ diquafosol tetrasodium, DE symptoms slightly improved. The BUT value improved from 0 to $8 \mathrm{~s}$, and no fluorescein staining was observed (A, D). Functional VA (E) and accommodative parameters improved I month after treatment (F).

Abbreviations: DE, dry eye; BUT, break-up time; Fk-map, frequency of kinetic reaction-map; HFC, high-frequency component; VA, visual acuity.

be an important indicator of "ocular fatigue", not only in the young, but also in elderly patients.

We observed three patients with no symptomatic improvement after DE treatment, although tear stability, visual function, and AMF were improved in these patients. These results suggest that a combination of tear instability and accommodative stress may be a trigger not only for DE symptom provocation, but also for corneal pain sensitivity. ${ }^{20}$

The AMF is a difficult phenomenon; thus, further discussion is necessary to understand it. It has been reported that several variables affect AMF, such as age, ${ }^{21}$ pupil diameter, ${ }^{11}$ astigmatism, ${ }^{22}$ and cardiopulmonary signals. ${ }^{23,24}$ Furthermore, while the LFC is thought to play a role in the control of the accommodative response and is produced by the optical fluctuation in the crystalline lens, ${ }^{25}$ there is no common consensus regarding HFC. ${ }^{23,24}$ Some reports have noted a correlation between HFC and the cardiopulmonary system. ${ }^{23}$ However, it is unknown how the cardiopulmonary system is related to the appearance of HFC and the symptoms of short BUT DE. There might be a relation between ciliary muscle spasms and arterial pulse.

There are a couple of limitations in this study. Since it is a cross-sectional study, there is not enough evidence to conclude the causal relationship in the association between short BUT DE and AMF. Moreover, this study did not have a control group with subjects without short BUT values. The other limitation is that the LFC values were not evaluated. Besides, we 


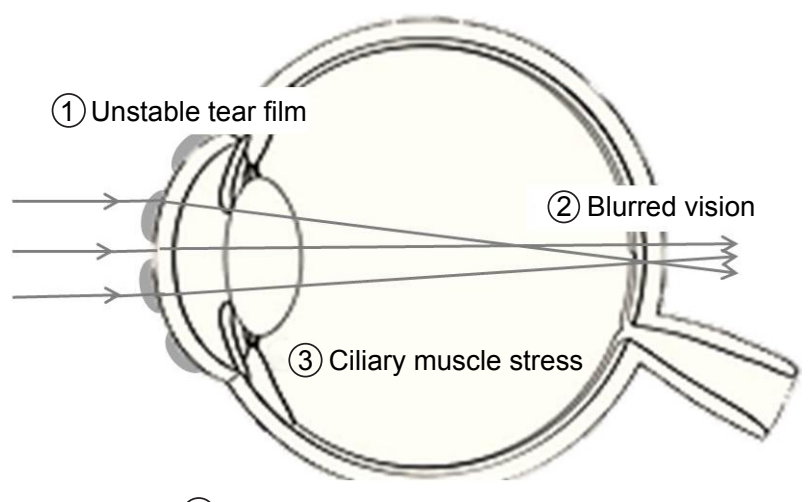

(4) AMFs DE symptoms

Sagittal optical structure

Figure 4 The mechanism of occurrence of dry eye symptoms. Image defocus due to tear instability represents visual impairment.

Note: Blurring may possibly cause ciliary muscle spasms, which eventually induce AMF and cause DE symptoms including ocular fatigue.

Abbreviations: AMF, accommodative microfluctuation; DE, dry eye.

did not assess some factors such as pupil diameter, astigmatism, and cardiopulmonary signals, which may affect AMF values, as mentioned above. Further studies with a larger cohort may be necessary to confirm the results related to the AMF.

In conclusion, this study proposed for the first time an association between AMF and the symptoms of short BUT DE. Our results showing an improvement in HFC1, functional VA, and BUT value after DE treatment support the idea that an unstable tear film may induce AMF and ocular fatigue in short BUT DE.

\section{Authors' contributions}

Conception and design of the study: M Kaido, K Tsubota; analysis and interpretation of results: $M$ Kaido; manuscript preparation: M Kaido; critical review of the article: M Kaido, M Kawashima, R Ishida, K Tsubota; data acquisition: M Kaido, M Kawashima; provision of materials, patients, or resources: M Kaido, R Ishida; statistical expertise, literature searches: M Kaido; administrative, technical, or logistic support: M Kaido, K Tsubota. All authors contributed toward data analysis, drafting and critically revising the paper and agree to be accountable for all aspects of the work.

\section{Disclosure}

Kowa has supplied grant money for research unrelated to this study to the Department of Ophthalmology of Keio University School of Medicine.

The funding organization had no role in the design or conduct of this research study. Doctors Kazuo Tsubota and Minako Kaido hold patent rights for the method and the apparatus used for the measure of functional visual acuity
(US patent no: 7470026). The other authors have no conflicts of interest to report.

\section{References}

1. Schein OD, Muñoz B, Tielsch JM, Bandeen-Roche K, West S. Prevalence of dry eye among the elderly. Am J Ophthalmol. 1997;124(6): 723-728.

2. Lin PY, Tsai SY, Cheng CY, Liu JH, Chou P, Hsu WM. Prevalence of dry eye among an elderly Chinese population in Taiwan: the Shihpai eye study. Ophthalmology. 2003;110(6):1096-1101.

3. Schaumberg DA, Sullivan DA, Buring JE, Dana MR. Prevalence of dry eye syndrome among US women. Am J Ophthalmol. 2003;136(2): 318-326.

4. Uchino M, Yokoi N, Uchino Y, et al. Prevalence of dry eye disease and its risk factors in visual display terminal users: the Osaka study. Am J Ophthalmol. 2013;156(4):759-766.

5. Kaido M, Uchino M, Yokoi N, et al. Dry-eye screening by using a functional visual acuity measurement system: the Osaka study. Invest Ophthalmol Vis Sci. 2014;55(5):3275-3281.

6. Wu H, Wang Y, Dong N, et al. Meibomian gland dysfunction determines the severity of the dry eye conditions in visual display terminal workers. PLoS One. 2014;9(8):e105575.

7. Toda I, Shimazaki J, Tsubota K. Dry eye with only decreased tear break-up time is sometimes associated with allergic conjunctivitis. Ophthalmology. 1995;102(2):302-309.

8. Yokoi N, Uchino M, Uchino Y, et al. Importance of tear film instability in dry eye disease in office workers using visual display terminals: the Osaka study. Am J Ophthalmol. 2015;159(4):748-754.

9. Toda I, Fujishima H, Tsubota K. Ocular fatigue is the major symptom of dry eye. Acta Ophthalmol (Copenh). 1993;71(3):347-352.

10. Owens DA, Wolf-Kelly K. Near work, visual fatigue, and variations of oculomotor tonus. Invest Ophthalmol Vis Sci. 1987;28(4):743-749.

11. Campbell FW, Robson JG, Westheimer G. Fluctuations of accommodation under steady viewing conditions. J Physiol. 1959;145(3): 579-594.

12. Kajita M, Ono M, Suzuki S, Kato K. Accommodative microfluctuation in asthenopia caused by accommodative spasm. Fukushima J Med Sci. 2001;47(1):13-20.

13. Maeda F, Tabuchi A, Kani K, Kawamoto K, Yoneda T, Yamashita T. Influence of three-dimensional image viewing on visual function. Jpn J Ophthalmol. 2011;55(3):175-182.

14. Jeng WD, Ouyang Y, Huang TW, et al. Research of accommodative microfluctuations caused by visual fatigue based on liquid crystal and laser displays. Appl Opt. 2014;53(29):H76-H84.

15. van Bijsterveld OP. Diagnostic tests in the Sicca syndrome. Arch Ophthalmol. 1969;82(1):10-14

16. Findl O, Kiss B, Petternel V, et al. Intraocular lens movement caused by ciliary muscle contraction. J Cataract Refract Surg. 2003; 29(4):669-676.

17. Kaido M, Ishida R, Dogru M, et al. Visual function changes after punctal occlusion with the treatment of short BUT type of dry eye. Cornea. 2012;31(9):1009-1013.

18. Koh S, Maeda N, Horohara Y, et al. Serial measurements of higher-order aberrations after blinking in patients with dry eye. Invest Ophthalmol Vis Sci. 2008;49(1):133-138.

19. Yamamoto Y, Yokoi N, Higashihara H, et al. [Clinical characteristics of short tear film breakup time (BUT)-type dry eye]. Nihon Ganka Gakkai Zasshi. 2012;116(12):1137-1143. Japanese.

20. Rosenthal P, Borsook D. The corneal pain system. Part I: the missing piece of the dry eye puzzle. Ocul Surf. 2012;10(1):2-14.

21. Toshida K, Okuyama F, Tokoro T. Influence of the accommodative stimulus and aging on the accommodative microfluctuations. Optom Vis Sci. 1998;75(3):221-226.

22. Stark LR, Strang NC, Atchison DA. Dynamic accommodation response in the presence of astigmatism. J Opt Soc Am. 2003;20(12):2228-2236. 
23. Winn B, Pugh JR, Gilmartin B, Owens H. Arterial pulse modulates steadystate ocular accommodation. Curr Eye Res. 1990;9(10):971-975.

24. Collins M, Davis B, Wood J. Microfluctuations of steady-state accommodation and the cardiopulmonary system. Vision Res. 1995;35(17): 2491-2502.
25. Charman WN, Heron G. Fluctuations in accommodation: a review. Ophthalmic Physiol Opt. 1988;8(2):153-164.

\section{Publish your work in this journal}

Clinical Ophthalmology is an international, peer-reviewed journal covering all subspecialties within ophthalmology. Key topics include: Optometry; Visual science; Pharmacology and drug therapy in eye diseases; Basic Sciences; Primary and Secondary eye care; Patien Safety and Quality of Care Improvements. This journal is indexed on

Submit your manuscript here: http://www.dovepress.com/clinical-ophthalmology-journal
Dovepress

PubMed Central and CAS, and is the official journal of The Society of Clinical Ophthalmology (SCO). The manuscript management system is completely online and includes a very quick and fair peer-review system, which is all easy to use. Visit http://www.dovepress.com/ testimonials.php to read real quotes from published authors. 